microemboli; 2) alterations in blood flow, distribution, and pressure; and 3) neuropsychological effects of neuroleptic drugs, anxiety, pain, and postoperative isolation. Some protection from cerebral injury is provided by hypothermia and drugs that decrease cerebral metabolism.

\title{
CEREBRAL BLOOD VOLUME AFTER BIRTH ASPHYXIA
}

Cerebral hemodynamics and oxygenation in 18 perinatally asphyxiated neonates were compared with 13 healthy controls using near-infrared spectroscopy (NIRS) at the Dept of Pediatrics, University Hospital of Leiden, The Netherlands. Cerebral blood volume (CBV) in the first 12 hours of life was decreased in all of 9 severely asphyxiated neonates who subsequently developed neurologic abnormalities. This decrease in CBV was associated with a drop in $\mathrm{HbO}_{2}$ and cytochrome oxidase. All patients showed stable CBV and enzyme patterns at 12 to 24 hours,. The findings suggest that posthypoxicischemic reperfusion injury of the brain occurs during early neonatal life after severe birth asphyxia. (van Bel F et al. Changes in cerebral hemodynamics and oxygenation in the first 24 hours after birth asphyxia. Pediatrics Sept 1993;92:365-372). (Reprints: Frank van Bel MD,PhD, University Hospital Leiden, Neonatal Unit, Bldg 35, PO Box 9600, 2300 RC Leiden, The Netherlands).

COMMENT. The decrease in cerebral CBV, oxyhemoglobin, and cytochrome oxidase during the first 12 hours of life are indicators of decreased cerebral perfusion and oxygenation. The authors propose a possible relation between a decreased CBV and adverse neurologic outcome, suggesting a relation between cerebral hypoperfusion and brain tissue damage in severely asphyxiated neonates. NIRS may be used to monitor changes in $\mathrm{CBV}$ of neonates.

\section{CEREBRAL ULTRASOUND AND NEURODEVELOPMENT}

The relations between lesions detected by ultrasound brain-imaging and neurodevelopmental outcome at 8 years in a cohort of 206 very preterm infants were studied at the Department of Paediatrics, University College and Middlesex School of Medicine, London. Scans were normal at discharge from hospital in 112 (54\%), and of these, 4\% developed major, disabling impairment and $22 \%$ had minor impairments. Uncomplicated periventricular hemorrhage (PVH) was diagnosed in 55 (27\%), ventricular dilatation in $21(10 \%)$, hydrocephalus developed in 5, and cerebral atrophy was found in $13(6 \%)$. Disabling neurodevelopmental impairments, including epilepsy, mental retardation and cerebral palsy, were documented in 25 children at follow-up (12\%). Ventricular dilatation and cerebral atrophy were significant independent predictors of impairment of neurodevelopment, of school performance or achievement, and of the WISC-R Full-scale and Performance subscale IQs. (Roth SC, Reynolds EOR et al. Relation between ultrasound 
appearance of the brain of very preterm infants and neurodevelopmental impairment at eight years. Dev Med Child Neurol Sept 1993; $\underline{35}$ :755-768). (Respond: Dr Simon C Roth, Dept Paediatrics, University College and Middlesex School of Medicine, The Rayne Institute, 5 University St, London WC1E 6JJ, England).

COMMENT. A normal ultrasound scan or a finding of uncomplicated periventricular hemorrhage in very preterm infants permits reassurance and the likelihood of a favorable developmental outcome. In contrast, infants with ventricular dilatation, hydrocephalus and cerebral atrophy have a poor prognosis. Learning difficulties experienced by some preterm infants may be caused by undetected hypoxic-ischemic injury.

Routine ultrasound screening in a multicenter study involving 15,151 pregnant women did not improve perinatal outcome when compared to the selective use of ultrasonography only for medical indications on the basis of clinical judgment (Ewigman BG et al. Effect of prenatal ultrasound screening on perinatal outcome. $\mathrm{N}$ Engl I Med Sept

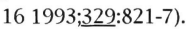

\section{CEREBRAL HYPOPERFUSION IN MYOTONIC DYSTROPHY}

Cerebral blood flow and perfusion measured by SPECT (Xenon and TcHMPAO), MRI findings, and neuropsychological function in 22 patients with myotonic dydtrophy (MD) were studied at the Departments of Neurology, Radiology, Psychiatry, and Pediatrics, Harbor-UCLA Medical Center, Torrance, CA. Results in 8 patients with maternal (mMD) inheritance were compared with those in 11 with paternal (pMD) inheritance, and in 10 normal controls. Patients with mMD had significantly lower scores on IQ tests and more extensive cerebral hypoperfusion when compared to those with pMD. Decreases in cerebral blood flow, most severe in frontal and temporoparietal association cortex, correlated strongly with lower scores on IQ tests. Patients with $\mathrm{mMD}$ had earlier onset of illness than the pMD group (means, 6.3 vs 32 yrs). Cerebral hypoperfusion patterns in the mMD patients were consistent with diffuse brain injury, whereas pMD patients showed only minor changes. (Chang L et al. Cerebral abnormalities in myotonic dystrophy. Cerebral blood flow, magnetic resonance imaging, and neuropsychological tests. Arch Neurol Sept 1993; $\underline{50}: 917-923)$. (Reprints: Dr Chang, Dept Neurology, Harbor-UCLA Medical Center, 1000 W Carson St, F-9, Torrance, CA 90509).

COMMENT. Mothers with myotonic dystrophy should be warned that their children would have a high probability of mental retardation. Hypoxic injury in utero is suggested as the likely explanation for the brain injury. Milder abnormalities in cerebral blood flow seen in the pMD group were attributed to possible sleep apnea, since many had hypersomnolence. 